\title{
Cost-Effectiveness of Carbon Ion Radiation Therapy for Skull Base Chordoma Utilizing Long-Term (10-Year) Outcome Data
}

\author{
TANJA SPRAVE ${ }^{1}$, VIVEK VERMA ${ }^{2}$, FLORIAN STERZING ${ }^{3}$, THOMAS BRUCKNER ${ }^{1,4}$, KATHARINA HEES $^{1,4,5}$, \\ BEATE LAND $^{6}$, OLIVER JÄKEL ${ }^{7,8}$, KLAUS HERFARTH ${ }^{1}$, JÜRGEN DEBUS ${ }^{1,9}$ and MATTHIAS UHL ${ }^{1}$ \\ ${ }^{1}$ Department of Radiation Oncology, University Hospital of Heidelberg, Heidelberg, Germany; \\ ${ }^{2}$ Department of Radiation Oncology, University of Nebraska Medical Center, Omaha, NE, U.S.A.; \\ ${ }^{3}$ Department of Radiation Oncology, Kempten Clinic, Kempten, Germany; \\ ${ }^{4}$ Department of Medical Biometry, University Hospital of Heidelberg, Heidelberg, Germany; \\ ${ }^{5}$ Department of Statistics, TU Dortmund University, Dortmund, Germany; \\ ${ }^{6}$ Baden-Wuerttemberg Cooperative State University (DHBW) Faculty of Economics, Mannheim, Germany; \\ ${ }^{7}$ Heidelberg Ion-Beam Therapy Center, Heidelberg, Germany; \\ ${ }^{8}$ German Cancer Research Center, Div. Medical Physics in Radiation Oncology, Heidelberg, Germany; \\ ${ }^{9}$ Clinical Cooperation Unit Radiation Oncology, German Cancer Research Center (DKFZ), Heidelberg, Germany
}

\begin{abstract}
Background/Aim: Carbon ion radiotherapy (CIRT) offers high conformality and ability to dose-escalate skull base chordomas, with promising clinical data. However, it is an imperative measure to economically justify the use of such high-priced new technologies. Herein, we investigated the cost-effectiveness of CIRT compared to photon radiotherapy (PRT) using 10-year outcome data extrapolated to a 34-year time frame. Materials and Methods: Data regarding costs of PRT, as well as 10-year outcomes were obtained from published sources. Corresponding figures for CIRT were acquired from institutional and published sources. Adjustment was made in order to compare both cost figures, including elimination of additional financing and follow-up, so that only direct costs of treatment and the cost of progression were compared between both modalities. The incremental cost-effectiveness ratio (ICER) was calculated as the difference in cost between both modalities divided by the difference in 34-year qualityadjusted life-year (QALY) outcomes. The annual gross domestic product per capita cost-effectiveness threshold definition (as recommended by the WHO) was employed. Results: The total cost of a complete course of CIRT (20-22 fractions) was $€ 31,538.21$. After removal of financing and
\end{abstract}

Correspondence to: Tanja Sprave, MD, MBA, Department of Radiation Oncology, University Hospital of Heidelberg, Im Neuenheimer Feld 400, 69120 Heidelberg, Germany. Tel: +49 062215634689, e-mail: tanja.sprave@med.uni-heidelberg.de

Key Words: Carbon ion radiation therapy, chordoma, costeffectiveness, health care policy, radiation therapy. follow-up costs, the adjusted direct cost of CIRT utilized for comparison was $€ 18,957.78$. In a previous publication, the cost of PRT was $€ 4,700.00$. ICERs were based upon these direct cost figures and the average of reported 10-year progression-free survival (PFS) values with PRT (41.1\%) and CIRT (54\%), as well as gained PFS years (10.66 years CIRT, 8.58 years PRT). QALYs were 6.65 for photon RT and 8.26 for CIRT, a difference of 1.61 discounted lifetime QALYs for patients treated with CIRT. The overall ICER was $€ 8,855.76 / Q A L Y$. If the cost of progression/recurrence treated with imatinib were included into the calculation, the total ICER was $€ 170.61 / Q A L Y$. Conclusion: CIRT is a highly cost-effective option to treat chordoma.

Although chordomas are rare tumors, their propensity for aggressive local growth and re-growth can be a substantial challenge for oncologic therapy $(1,2)$. Management focuses on surgical resection followed by postoperative radiotherapy (RT); however, owing to anatomical factors and invasion patterns, complete resection is often not possible (3). Because a notable prognostic factor in this population is local control (LC), there is a clear necessity for doseescalation (especially if gross disease is present) (4).

Technological innovations in RT have brought particle therapy to the forefront of cancer care. As pertaining to chordomas, there are several advantages to treatment with carbon ion RT (CIRT). CIRT is a highly conformal RT modality, which lends itself well to treating a neoplasm that has an intimate anatomic relationship with several organs-at-risk (OARs). This conformality is afforded by the physical properties of the massive carbon ion, leading to a Bragg peak of maximal dose deposition with minimal dose deposition 
proximal and distal to the target of interest. Together, this allows for the unique capability to dose-escalate high-risk and/or gross disease while maintaining OAR doses under tolerance levels. Additionally, radiobiologic properties of particle radiotherapy include a high relative biological effectiveness (RBE); this may be advantageous to enhance LC (especially in the doseescalated setting) and treat slower-growing tumors $(5,6)$. Moreover, CIRT allows for hypofractionation owing to the differential increase in RBE to tumor cells as compared to normal tissue at higher doses per fraction (7).

These theoretical notions have been buttressed by clinical data illustrating the high efficacy of CIRT for skull base chordomas; it has even been posited that photon therapy may be inherently insufficient to provide a long-term cure to this malignancy $(4,8$ 11). The value of CIRT in recurrent cases has also been studied, demonstrating appropriate utility in this setting as well (9). Taken together, recognizing the dearth of experiences with this new technology, CIRT has become the preferred option at most centers with both photon and particle treatment capabilities.

To this extent, what has been less well-studied is whether the efficacious outcomes observed with CIRT can translate into the economic realm. Though the cost-effectiveness (CE) of particle therapy is difficult to truly gauge, it is an imperative measure to justify the use of such high-priced new technologies $(12,13)$. A decade ago, a CE analysis was performed for CIRT in chordoma, displaying CIRT to be equally or more cost-effective as photons (14). However, as noted elsewhere, with the expansion of outcome data together with global economic changes and overall healthcare costs, the defined CE of particle therapy could change rapidly (12). Thus, we performed this contemporary analysis to address the CE of CIRT versus photons for the treatment of chordoma.

\section{Materials and Methods}

In order to provide an accurate comparison, the methodology of this analysis mirrored that published elsewhere, as much as possible (14). A notable exception herein was that CIRT was compared to the photon RT (PRT) modality of Gamma Knife stereotactic RT. Though it is acknowledged that conventionally-fractionated intensitymodulated RT is an often-performed photon modality for chordoma, Gamma Knife RT is indeed performed for select chordoma cases (15, 16). The principal reason for this methodological distinction was that the primary objective of this study, over the previous one, was to provide a CE estimate based on more long-term data. The only photon modality for which 10-year data exist is Gamma Knife RT $(15,16)$, and as such, cost values were estimated based on CE data of Gamma Knife RT for brain metastases in the same healthcare system as that of this study, during a contemporary time period (17). For PRT we used the pooled 5 and 10-year survival rates from the aforementioned publications $(15,16)$. Unfortunately, there were no standard deviations reported therein, so the numbers of patients were utilized as weights for the pooling.

Primary costs were estimated in a hypothetical population that had already undergone surgical resection and were to undergo adjuvant treatment using PRT or CIRT. Thus, surgical and postoperative costs were not needed to be calculated herein; though dissimilar from the prior analysis, this also eliminates another source of "economic bias" and/or miscalculation of all potential variables associated with surgery and postoperative course. In this study, a course of CIRT was designated per institutional protocol with 45 Gray-equivalents (GyE) in 15 fractions from the skull base to the bottom of the second cervical vertebra, followed by a 15-21 GyE boost (delivered in 5-7 fractions) to the initial extent of tumor.

Whereas PRT costs were described above, costs for CIRT were based on a systematic cost analysis of all tumor entities performed at a single CIRT facility from January to June 2013. This encompassed 328 patients treated with 5,743 CIRT fractions; of this population, 48 patients (15\%) had skull base neoplasm diagnoses. For each patient, institutional-perspective costs from initial consultation to follow-up were recorded, and financial data evaluated based on data provided by internal controlling. The result of this systematic cost analysis is displayed in Table I. Broadly, these encompassed costs (e.g. logistical and physician fees) of initial consultation, simulation (including costs of apparatus and imaging, including MRI fusion), treatment planning, each fraction of therapy (including on-treatment visits), and followup (including MRI imaging). In order to calculate direct costs of treatment alone, without including external financing and follow-up (as done for the photon comparator arm) (17), there were several adjustments performed. First, because these figures were presented with differential (50 and 100\%) amounts of financing, adjustment had to be made via removal of additional financing. This was done by subtracting the total costs of CIRT with $50 \%$ financing from those with $100 \%$ financing. The resulting value was then doubled to signify the total cost of financing, which was in turn subtracted from the $100 \%$ financed cost initially obtained. Costs of follow-up were then subtracted to form the direct cost of CIRT, without financing or follow-up so as to make an appropriate comparison with existing data of photon RT (17)

In order to calculate the incremental CE ratio (ICER), the direct cost of photon RT (17) was subtracted from the direct cost of CIRT as calculated above. This differential "cost" value was then divided by the "effectiveness" value. The latter referred to the 10 -year progression-free survival (PFS) of CIRT (9) subtracted by that of photon RT $(15,16)$, or alternatively the "gained PFS" between 5 and 10 year figures, mathematically extrapolated to a total time frame of 34 years, the remaining average life expectancy of the patients (average 40-50 years old) at treatment (18). The result was the time spent progression-free over the course of 34 years. This yielded an ICER value of Euros $(€)$ per year of PFS. In order to estimate quality-adjusted life years (QALYs), a publication was used that estimated the subjective quality of life for persons aged 40-50 years to be 77.5/100 (19). Therefore, we utilized a factor of 0.775 for the conversion of PFS years into QALYs. QALYs were discounted at $3 \%$ per year.

Subsequently, to address the cost of recurrence/progression, the discounted (3\% per year) cost of treatment for local progression was also determined and added to the direct cost, resulting in a set of ICERs that included this cost factor. For this study, the threshold to define CE was as recommended by the WHO. These are defined as three times the annual gross domestic product (GDP) per capita for "cost-effectiveness", and one time the annual GDP per capita for "high cost-effectiveness" (20). In 2013, the annual GDP per capita for Germany was $€ 35,045$ (21). Hence, thresholds for "cost effectiveness" and "high cost effectiveness" were $€ 105,135 / \mathrm{QALY}$ and $€ 35,045 / \mathrm{QALY}$, respectively. 


\section{Results}

Treatment costs. The total cost of a complete course of CIRT treatment (20-22 fractions), as initially calculated in Table I, was € $€ 31,538.21$ (including 50\% financing). Table II displays the adjustments made in order to calculate direct treatment costs. After removal of financing costs $(€ 23,694.66)$, the adjusted cost of CIRT was $€ 19,690.88$. When subtracting the $€ 733.10$ cost of follow-up (Table I), the adjusted direct cost of CIRT utilized for comparison was $€ 18,957.78$.

Cost-effectiveness. ICERs were based upon the direct cost figures in Table II, existing data on PFS $(15,16)$, as well as utilizing an extrapolation based method. The average life expectancy of persons 40-60 years old was 81 years (in 2013). Thus, since the average age at treatment of the three studies was 47 years, we evaluated a total time frame of 34 years post treatment (when on average all patients are deceased). Figure 1 displays the progression-free years of a hypothetical cohort for 34 years, by assuming a Weibull distribution for the survival curves. We estimated the parameters of the Weibull distribution by using the 5 and 10-year survival rates. From this, the area under the respective graph corresponded to the (average) total PFS years, which was 12.04 for photon RT and 14.70 for CIRT. After applying a $3 \%$ annual discount rate, figures were 8.58 and 10.66 PFS years, respectively. Hence, the discounted advantage of CIRT over photon RT was 2.08 PFS years. Utilizing the conversion factor from PFS years to QALYs of 0.775 as mentioned above, the QALYs were 6.65 for photon RT and 8.26 for CIRT. Thus, on average, patients treated with CIRT benefitted from 1.61 additional discounted lifetime QALYs in comparison to patients treated with photon RT. Hence, the ICER was $(€ 18,957.78$ $€ 4,700.00) / 1.61$ gained QALYs, totaling $€ 8,855.76 / \mathrm{QALY}$, which is well below a willingness-to-pay threshold of $€ 105,135 / \mathrm{Q} A L Y$, and also under the "high cost effectiveness" cutoff of $€ 35,045 /$ QALY.

Cost of progression/recurrence. Assuming that there was no role for repeat irradiation or neurosurgical intervention at recurrence, the cost of recurrence was calculated using a promising systemic agent, imatinib (22-23). Administered for two years, with a cost of $€ 280 /$ daily dose (400 mg twice daily at roughly $€ 140 / 400 \mathrm{mg}$ ), an expected total cost is $€ 204,400$ (24). Utilizing a 3\% annual discount rate, the discounted cost of imatinib for photon RT is $€ 148,319.07$ and for CIRT $€ 134,335.97$.

After removal of financing and follow-up, the total cost of CIRT was $€ 18,957.78$ (direct cost) plus $€ 134,335.97$ (discounted cost of imatinib), summing to $€ 153,293.75$. Analogously, costs for photon RT were $€ 4,700.00$ and
Table I. Detailed description of costs associated with a complete course of CIRT for skull base chordoma with $50 \%$ financing.

\begin{tabular}{lr}
\hline Parameter & Costs (Euros) \\
\hline Initial consultation & \\
Logistic, technical and physician feers & 85.47 \\
Simulation & 70.29 \\
Logistic, technical and physician feers & 200.00 \\
Aparatus (e.g. mask, IV contrast & 683.60 \\
Imaging (CT with MRI fusion) & 953.89 \\
Total & 146.28 \\
Treatment planning & \\
Logistic, technical and physician feers & $28,532.15$ \\
Treatment (20-22 fractions) & $1,071.32$ \\
RT delivery and treatment room use & $29,619.47$ \\
Logistic, technical and physician feers & 74.50 \\
Total & 658.60 \\
Follow up & 733.10 \\
Logistic, technical and physician feers & \\
Imaging (CT or MRI) & $31,538.21$ \\
\hline Total & \\
\hline Total costs of CIRT treatment & \\
\hline
\end{tabular}

CIRT, Carbon ion radiation therapy; IV, intravenous; CT, computed tomography; MRI, magnetic resonance imaging; RT, radiation therapy.

Table II. Adjustment of costs performed in this investigation.

\begin{tabular}{lc}
\hline Parameter & Costs (Euros) \\
\hline Direct PRP cost without financing or follow up & $4,700.00$ \\
Total CIRT cost with 50 \% financing & $31,583.21$ \\
Total CIRT cost with 100\% financing & $43,385.54$ \\
Total financing costs & $23,694.66$ \\
Direct CIRT cost without financing & $19,690.88$ \\
Direct CIRT cost without financing or follow up & $18,957.78$ \\
\hline
\end{tabular}

PRT, Photon radiation therapy; CIRT, carbon ion radiation therapy.

$€ 148,319.07$, summing $€ 153,019.07$. This led to an ICER of $€ 274.68 / 1.61 \mathrm{QALY} s=€ 170.61 / \mathrm{QALY}$.

Sensitivity analysis. We investigated the change in ICER under an uncertainty of $\pm 10 \%$ for the cost variables. For the 10 -year survival rate of CIRT, we used the $95 \%$ confidence interval (CI) (0.43 to 0.65$)$, and analyzed the change in ICER when utilizing the lower and upper bounds of the CI. Unfortunately, there were no standard deviations or confidence intervals reported for the 10-year survival rates in photon publications $(15,16)$, so we used a deviance of $\pm 10 \%$ for our pooled survival rate for the sensitivity analyses (approximately the same deviance as was used for CIRT from the $\mathrm{CI}$ ). The results of the sensitivity analysis are shown in Figure 2. The $\mathrm{x}$-axis shows the change in ICER compared to the value calculated in the results, which was $€ 8,855.76 / \mathrm{QALY}$, on a $\log$ scale. 


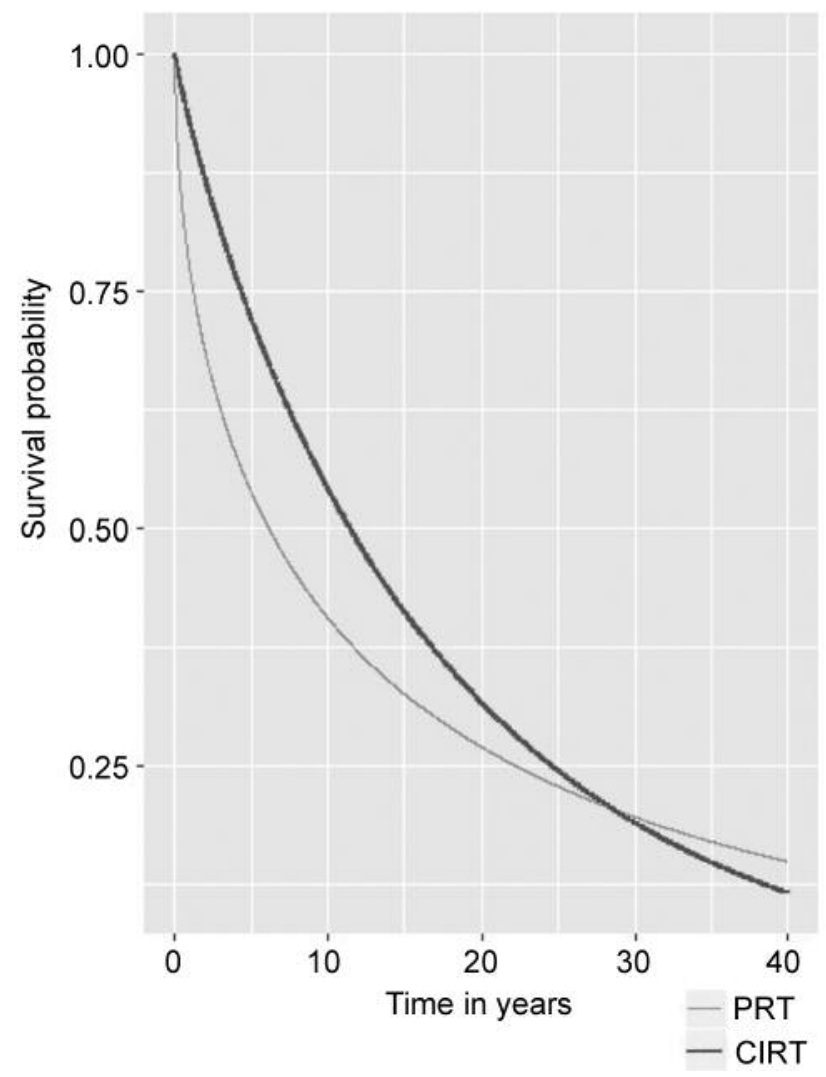

Figure 1. Weibull distribution.

\section{Discussion}

Economic analyses of emerging but largely unproven technologies such as CIRT are an essential adjunct to clinical studies of safety and efficacy. This contemporary CE investigation demonstrated that CIRT is an economically feasible alternative to PRT. Nevertheless, further work must corroborate the findings herein, especially because the inherent nature of $\mathrm{CE}$ studies necessitates non-uniform generalizability as well as several design biases that are present in any CE analysis (12).

The results of this study were driven by expected outcome benefits in the population receiving CIRT over PRT. As such, although this investigation did not utilize a modeling approach (wherein toxicities are accounted for), the fact that favorable CE figures were observed despite some level of anticipated toxicity (and thus cost) reduction, is noteworthy. In this study, lack of modeling and quality of life information are largely the result of very few data for this rare tumor; moreover, many high-quality investigations incorporate neither simulation models, quality of life data, nor sensitivity analyses (25-26). Others may utilize such methodologies, but

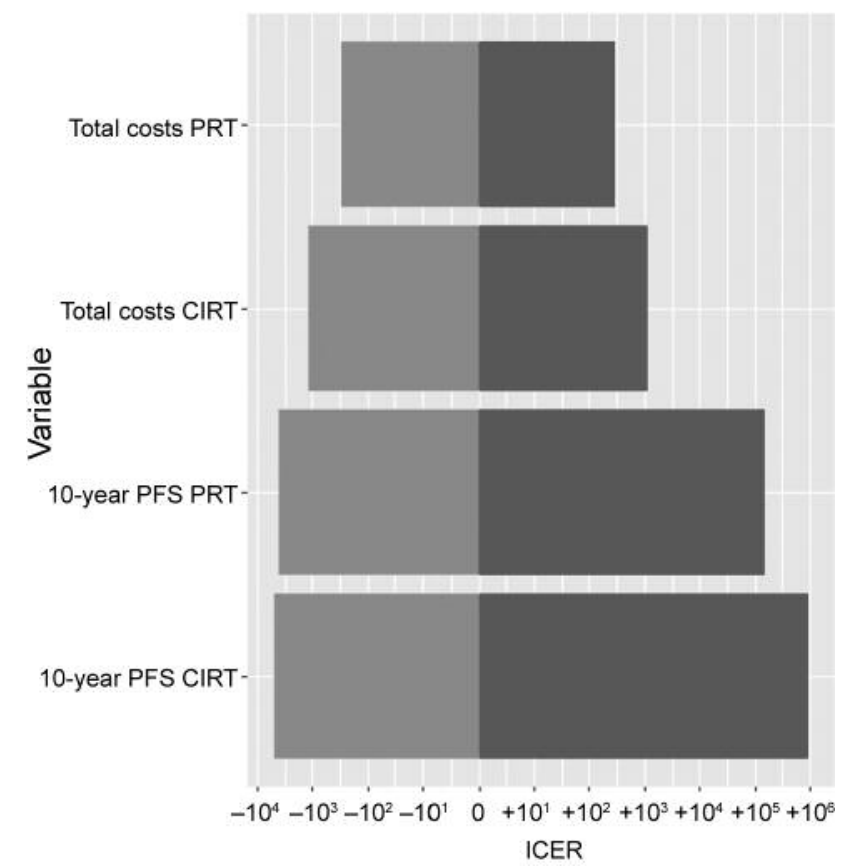

Figure 2. Sensitivity analysis.

have insufficient clinical data to back up conclusions (27). Taken together, although no CE study can come close to accounting for all possible costs (e.g. operational/capital investment costs, the "costs" of death, etc.), the result of such investigations is to merely provide a rough approximation of the $\mathrm{CE}$ between two modalities of cancer care.

It is important to understand that $\mathrm{CE}$ is a dynamically changing entity, especially in the realm of rapidly-evolving technologies such as CIRT and particle therapy in general. It has been posited that over the next decade, treatment costs for particle therapy could drop by a very substantial $20 \%$ (28). As evidence, reimbursements for proton therapy have even decreased in more recent times compared to the past (30). Other sources of economic change that may limit generalizability of this work include the status of the global economy. Few CE studies account for inflation, for example, and thus, applicability to conditions even a decade ago (i.e. the difference between this study and its predecessor) is limited. Accordingly, these data may not be applicable to other economic systems such as in the United States. Taken together, however, because accrual of clinical and toxicity data for CIRT is just beginning (with several ongoing phase II/III trials in several malignancies), a clearer economic picture will likely be available in the future.

These data were noticeably limited by several factors, such as in any CE study. First, the lack of CIs in prior publications $(15,16)$ has been mentioned above. Owing to a lack of 10- 
year PFS data in the CIRT cohort, we opted to roughly equate 10-year LC with 10-year PFS because the rate of distant disease in chordoma is predictably low; though this is theoretically sound and consistent with other work (14), it may be a source of error. Furthermore, it is a welldocumented shortcoming of most CE analyses that outcome data (utilized for calculation of $\mathrm{CE}$ ) is often based on 1 or a few largely retrospective studies, and this report was no exception (12). Moreover, another limitation was that the photon RT arm was represented by Gamma Knife RT data, which was necessary owing to Gamma Knife series being the only source of 10-year photon data. Because the primary objective of this study was to utilize long-term data as a basis for $\mathrm{CE}$ (hence the novelty over the prior CE study), the Gamma Knife data was utilized.

Furthermore, the data were limited insofar as it only covered a time frame of 10 years. After this time, a substantial amount of patients were still progression free. Therefore we extrapolated the results to cover a time frame that covered the entire remaining life expectancy of the average patient (34 years from treatment). This assumes that every patient experiences progression at some point in his/her life, which may or may not be the case. But it was still deemed to be the better approach, as we did not want to ignore the substantial benefits of both treatment methods after the 10-year time frame.

Our approach assumes that every patient experiences progression at some point in his/her life. Hence, substantial costs may be incurred for the treatment of disease progression. Our primary set of ICERs does not include such costs of progression, as there are several clinical approaches to manage this circumstance, each with very different cost profiles. Moreover, because the ICERs before progression were already highly cost-effective, a complete analysis of progression seemed relatively superfluous. However, it was considered unsatisfactory to ignore the cost of progression entirely. Therefore, we compiled a second set of ICERs, assuming treatment using imatinib, while not following-up regarding other alternative treatment methods. However, given the high rates of PFS reported with CIRT, including the costs of progression might likely reveal a more favorable economic profile for CIRT.

\section{Conclusion}

Based on this analysis, CIRT was found to be a highly costeffective option for the treatment of skull base chordoma. Further work should validate the conclusions presented herein, especially because the subjective interpretation of a modality being "cost-effective" is hence dependent on perspectives of a particular payer, patient, culture, and society.

\section{Conflicts of Interest}

J.D. is Chief Executive Officer of the Heidelberg Ion Beam Therapy Center (HIT). All remaining authors declare no conflicts of interest. This has never been presented/published before in any form.

\section{References}

1 Walcott BP, Nahed BV, Mohyeldin A, Coumans JV, Kahle KT and Ferreira MJ: Chordoma: current concepts, management, and future directions. Lancet Oncol 13: e69-76, 2012.

2 Smoll NR, Gautschi OP, Radovanovic I, Schaller K and Weber DC: Incidence and relative survival of chordomas: the standardized mortality ratio and the impact of chordomas on a population. Cancer 119: 2029-2037, 2013.

3 Tzortzidis F, Elahi F, Wright D, Natarajan SK and Sekhar LN: Patient outcome at long-term follow-up after aggressive microsurgical resection of cranial base chordomas. Neurosurgery 59: 230-237, 2006.

4 Schulz-Ertner D, Karger CP, Feuerhake A, Nikoghosyan A, Combs SE, Jäkel O, Edler L, Scholz $M$ and Debus J: Effectiveness of carbon ion radiotherapy in the treatment of skull-base chordomas. Int J Radiat Oncol Biol Phys 68: 449457, 2007.

5 Yamazaki H, Demizu Y, Okimoto T, Ogita M, Himei K, Nakamura S, Suzuki G, Yoshida K, Kotsuma T, Yoshioka Y and Oh R: Reirradiation for recurrent head and neck cancers using charged particle or photon radiotherapy. Strahlenther Onkol 193: 525-533, 2017.

6 Riechardt A, Karle B, Cordini D, Heufelder J, Budach V, Joussen AM and Gollrad $\mathrm{J}$ : Proton therapy of iris melanoma with 50 CGE: influence of target volume on clinical outcome. Strahlenther Onkol 193: 943-950, 2017.

7 Ando K, Koike S, Uzawa A, Takai N, Fukawa T, Furusawa Y, Aoki $\mathrm{M}$ and Miyato $\mathrm{Y}$ : Biological gain of carbon-ion radiotherapy for the early response of tumor growth delay and against early response of skin reaction in mice. J Radiat Res 46: 51-57, 2005.

8 Mizoe JE, Hasegawa A, Takagi R, Bessho H, Onda T and Tsujii $\mathrm{H}$ : Carbon ion radiotherapy for skull base chordoma. Skull Base 19: 219-224, 2009.

9 Uhl M, Mattke M, Welzel T, Roeder F, Oelmann J, Habl G, Jensen A, Ellerbrock M, Jäkel O, Haberer T, Herfarth K and Debus J: Highly effective treatment of skull base chordoma with carbon ion irradiation using a raster scan technique in 155 patients: first long-term results. Cancer 120: 3410-3417, 2014.

10 Fuller DB and Bloom JG: Radiotherapy for chordoma: Int J Radiat Oncol Biol Phys 15: 331-339, 1988.

11 Romero J, Cardenes A, la Torre A, Valcarcel F, Magallon R, Regueiro $\mathrm{C}$ and Aragon G: Chordoma: results of radiation therapy in eighteen patients. Radiother Oncol 29: 27-32, 1993.

12 Verma V, Mishra MV and Mehta MP: A systematic review of the cost and cost-effectiveness studies of proton radiotherapy. Cancer 122: 1483-1501, 2016.

13 Verma V, Shah C, Rwigema JC, Solberg T, Zhu X and Simone CB 2nd: Cost-comparativeness of proton versus photon therapy. Chin Clin Oncol 5: 56, 2016.

14 Jäkel O, Land B, Combs SE, Schulz-Ertner D and Debus J: On the cost-effectiveness of Carbon ion radiation therapy for skull base chordoma. Radiother Oncol 83: 133-138, 2007. 
15 Tamura T, Sato T, Kishida Y, Ichikawa M, Oda K, Ito E, Watanabe T, Sakuma $\mathrm{J}$ and Saito K: Outcome of clival chordomas after skull base surgeries with mean follow-up of 10 years. Fukushima J Med Sci 61: 131-140, 2015.

16 Boari N, Gagliardi F, Cavalli A, Gemma M, Ferrari L, Riva P and Mortini P: Skull base chordomas: clinical outcome in a consecutive series of 45 patients with long-term follow-up and evaluation of clinical and biological prognostic factors. J Neurosurg 125: 450-460, 2016.

17 Vuong DA, Rades D, van Eck AT, Horstmann GA and Busse R: Comparing the cost-effectiveness of two brain metastasis treatment modalities from a payer's perspective: Stereotactic radiosurgery versus surgical resection. Clin Neurol Neurosurg 115: 276-284, 2013.

18 DE Statis. Statistisches Bundesamt. Lebenserwartung in Deutschland. https://www.destatis.de/DE/ZahlenFakten/Gesell schaftStaat/Bevoelkerung/Sterbefaelle/Sterbefaelle.html\#Tabelle n (Accessed June 6, 2018).

19 Hinz A, Klaiberg A, Brahler E and Konig HH: Der Lebensqualitatsfragebogen EQ-5D: Modelle und Normwerte fur die Allgemeinbevolkerung. Psychotherapie Psychosomatik Medizinische Psychologie 56(02): 42-48, 2006.

20 World Health Organization. 2002 Health Report. http://www. who.int/whr/2002/en/whr02_en.pdf (Accessed March 8, 2017).

21 Statistisches Landesamt Baden-Württemberg, Wirtschaftskraft. https://www.statistik-bw.de/GesamtwBranchen/VGR/VW_ wirtschaftskraft.jsp/ (Accessed June 6, 2018).

22 Stacchiotti S, Sommer J and Chordoma Global Consensus Group: Building a global consensus approach to chordoma: a position paper from the medical and patient community. Lancet Oncol 16: e71-83, 2015.

23 Stacchiotti S, Longhi A, Ferraresi V, Grignani G, Comandone A, Stupp R, Bertuzzi A, Tamborini E, Pilotti S, Messina A, Spreafico C, Gronchi A, Amore P, Vinaccia V and Casali PG: Phase II study of imatinib in advanced chordoma. J Clin Oncol 30: 914-920, 2012.
24 Schwabe U and Paffrath D (eds): Arzneiverordnungs-Report 2015: Aktuelle Zahlen, Kosten, Trends, und Kommentare. Heidelberg, Germany, Springer-Verlag, pp. 854, 2015.

$25 \mathrm{Yu}$ JB, Soulos PR, Herrin J, Cramer LD, Potosky AL, Roberts $\mathrm{KB}$ and Gross $\mathrm{CP}$ : Proton versus intensity-modulated radiotherapy for prostate cancer: patterns of care and early toxicity. J Natl Cancer Inst 105: 25-32, 2013.

26 Aizer AA, Gu X, Chen MH, Choueiri TK, Martin NE, Efstathiou JA, Hyatt AS, Graham PL, Trinh QD, Hu JC and Nguyen PL: Cost implications and complications of overtreatment of lowrisk prostate cancer in the United States. J Natl Compr Canc Netw 13: 61-68, 2015.

27 Taghian AG, Kozak KR, Katz A, Adams J, Lu HM, Powell SN and DeLaney TF: Accelerated partial breast irradiation using proton beams: initial dosimetric experience. Int J Radiat Oncol Biol Phys 65: 1404-1410, 2006.

28 Peeters A, Grutters JPC, Pijls-Johannesma M, Reimoser S, De Ruysscher D, Severens JL, Joore MA and Lambin P: How costly is particle therapy? Cost analysis of external beam radiotherapy with carbon-ions, protons, and photons. Radiother Oncol 95: 45$53,2010$.

29 Goitein $\mathrm{M}$ and Jermann M: The relative costs of proton and $\mathrm{x}$-ray radiation therapy: Clin Oncol (R Coll Radiol) 15: 37-50, 2003.

30 Jarosek S, Elliott S and Virnig BA: Proton beam radiotherapy in the US Medicare population: growth in use between 2006 and 2009: data points 10.2012 May 7. In: Data Points Publication Series. Rockville, MD: US Agency for Healthcare Research and Quality; 2011. Available at: http://www.ncbi.nlm.nih.gov/ books/NBK97147/ (Accessed December 26, 2016).

Received May 21, 2018

Revised June 16, 2018

Accepted June 22, 2018 